\title{
AN INTEGRAL EQUATION FOR THE DISTRIBUTION OF THE FIRST EXIT TIME OF A REFLECTED BROWNIAN MOTION
}

\author{
VICTOR DE-LA-PEÑA ${ }^{\otimes 1}$, GERARDO HERNÁNDEZ-DEL-VALLE ${ }^{2}$ and \\ CARLOS G. PACHECO-GONZÁLEZ ${ }^{3}$
}

(Received 15 March, 2009; revised 31 March, 2009)

\begin{abstract}
Reflected Brownian motion is used in areas such as physiology, electrochemistry and nuclear magnetic resonance. We study the first-passage-time problem of this process which is relevant in applications; specifically, we find a Volterra integral equation for the distribution of the first time that a reflected Brownian motion reaches a nondecreasing barrier. Additionally, we note how a numerical procedure can be used to solve the integral equation.
\end{abstract}

2000 Mathematics subject classification: primary 60G07; secondary 60G17, 45D05, $65 \mathrm{D} 15$.

Keywords and phrases: reflected Brownian motion, first passage time, Volterra integral equation.

\section{Introduction}

Reflected Brownian motion has received considerable attention due to its capacity to model several real phenomena in physics, biology and chemistry; see Grebenkov [12], where several applications are mentioned. It is known that this model is very suitable for modelling the interaction between a particle diffusing in a medium and an interface, where the particle may suffer a "reflection" (see for example [12, 18]). First-passagetime problems are important in such applications (see for instance Levitz et al. [18]), which motivates us to study the distribution of the time when the process surpasses an increasing varying level/barrier, that is, the time when the particle interacting with the

\footnotetext{
${ }^{1}$ Statistics Department, Columbia University, 1255 Amsterdam Ave. Mail Code 4403, New York, NY, USA; e-mail:vp@stat.columbia.edu.

${ }^{2}$ Statistics Department, Columbia University, USA; e-mail: gerardo@stat.columbia.edu.

${ }^{3}$ Departamento de Matematicas, CINVESTAV-IPN, A. Postal 14-740, Mexico D.F. 07000, Mexico, USA; e-mail: cpacheco@math.cinvestav.mx.

(C) Australian Mathematical Society 2009, Serial-fee code 1446-1811/2009\$16.00
} 
interface reaches a specific level in the medium (separated from the interface). Hence, we concentrate on finding the distribution of

$$
T:=\inf \left\{t:\left|B_{t}\right|=f(t)\right\},
$$

where $B=\left\{B_{t}: t \geq 0\right\}$ is a standard Brownian motion (BM) and $f(t)$ is a nondecreasing function with $f(0)>0$.

However, this issue can be seen as a particular case of the first time that the BM exits a moving range. Let $f(t)$ and $g(t)$ be two positive functions such that $f(0)>0$ and $g(0)>0$ (that is, the BM starts in between the barriers). The general probabilistic question is to find the distribution of

$$
T^{*}:=\inf \left\{t: B_{t} \notin(-g(t), f(t))\right\} .
$$

There are works in the literature concerning first-exit-time problems from varying regions, with single and double barriers. Considering a single barrier, see for example Ricciardi et al. [24], Peskir [22], De-la-Peña and Hernández-del-Valle [10], Darling and Siegert [7].

Regarding two-sided barriers, as in (1.1), there has been also a lot of interest, mainly from a theoretical point of view. Lifshits and Shi [21], address the tail behaviour of the exit-time distribution from parabolic domains of a planar Brownian motion, see also [1, 19, 20]. Deblassie [9] studies the probability that a Brownian motion (with dimension higher than one) remains in what he calls horn-shaped domains. Other related works are $[5,8,19]$.

Notice that reflected Brownian motion is a one-dimensional Bessel process; in Betensky [3] first-passage issues for Bessel processes are addressed.

Here we focus on characterizing the distribution of $T$.

The paper is organized as follows. In Section 2 we give preliminary results. In Section 3 we find an integral equation for the first exit time distribution of the reflected Brownian motion, and in Section 4 we state the integral equation for the density. Finally we use a numerical method to solve integral equations. We also give conclusions and mention some open problems.

\section{Hitting times of a Brownian motion}

To start studying exit-times distribution, we define common variables that we use. Process $B$ will be Brownian motion (BM) throughout the paper. The following hitting times are of interest:

$$
T^{f}:=\inf \left\{t \geq 0: B_{t}=f(t)\right\}, \quad T_{-g}:=\inf \left\{t \geq 0: B_{t}=-g(t)\right\},
$$

where $f$ and $g$ are two functions such that $f(0)>0$ and $g(0)>0$.

The first fact we have to notice is that $T_{-f} \stackrel{d}{=} T^{f}$, by symmetry of the BM ( $\stackrel{d}{=}$ stands for equality in distribution). We readily see that the exit time $T$, defined in (1.1), satisfies $T=T^{f} \wedge T_{-g}$. So, the exit time is the first time $B$ hits any of the barriers. 
The following cases are known (refer to [11, 14-16, 23]). If $f(t)=a$ for all $t \geq 0$, the density $\varphi_{a}(s)$ of $T^{f}$ is given by

$$
\varphi_{a}(s)=\frac{a}{\sqrt{2 \pi s^{3}}} \exp \left\{-\frac{a^{2}}{2 s}\right\}, \quad s \geq 0 .
$$

If $f(t)=c t+a$, with $a>0$, the density becomes

$$
\varphi_{f}(s)=\frac{a}{\sqrt{2 \pi s^{3}}} \exp \left\{-\frac{(c s+a)^{2}}{2 s}\right\}, \quad t \geq 0 .
$$

For the case of constant barriers $f(t)=g(t)=a$, it is known that the Laplace transform of $T$ [23, Proposition 2.3.7] is

$$
E\left(e^{-\theta T}\right)=\frac{1}{\cosh (a \sqrt{2 \theta})} .
$$

Thus, the density is given by the inverse function. In this paper, we shall work with a more general form.

Proposition 2.1 ([4, Page 172]). Consider a Brownian motion B starting at $x$. The density of $T$, the first time $B$ exits $[-a, b]$ with $x \in[-a, b]$, is given by

$$
P(T \in d t)=s s_{(b-x, b+a)}(t)+s s_{(x+a, b+a)}(t),
$$

where

$$
s s_{(u, v)}(t)=\sum_{k=-\infty}^{\infty} \frac{v-u+2 k v}{\sqrt{2 \pi t^{3}}} e^{-(v-u+2 k v)^{2} /(2 t)} .
$$

Furthermore, the expected value of $T$ is $E(T)=(x+a)(b-x)$ [23, Exercise 2.3.11].

We denote by $\varphi_{x}^{(-a, b)}(t)$ the density function $P(T \in d t)$ in (2.2).

\section{Integral equation}

When the functions $f$ and $g$ in (1.1) are nondecreasing, $T$ is said to be the first time that $B$ exits the "horn-shape" $\{(-g(t), f(t)): t \geq 0\}$. In this section, we shall derive an integral equation to compute the distribution of the first time a BM leaves a region determined by reflective barriers, that is, when $g=f$. First we need some notation.

3.1. Notation Let $f$ and $g$ be two positive functions.

- We denote by $T^{f}$ the first time a BM hits $f$, and $T_{-g}$ when it hits $-g$. The notation is the same when $f$ and $g$ are constants, namely $T^{b}$ or $T_{-a}$.

- We denote by $T_{-g}^{f}(x)$ the first time a BM starting at $x$ hits $f$ or $-g$, where $x \in[-g(0), f(0)]$. So $T_{-a}^{b}(x)$ denotes the hitting time when the barriers are constants. When there is no ambiguity we write $T_{-g}^{f}(0)$ as $T$. 
- The distribution of $T_{-g}^{f}(x)$ is denoted by $\Phi_{x}^{(-g, f)}(t):=P\left(T_{-g}^{f}(x)<t\right)$, or $\Phi(t)$ when $x=0$.

Notice that if the barriers are constants, the distribution is known, and the density is given by $\varphi_{x}^{(-a, b)}(u)$ in (2.2). For example, if we fix $t$ and $s$, then $\varphi_{x}^{(-g(t), f(s))}(u)$ denotes the density of the first time a BM hits either of the fixed points $-g(t)$ or $f(s)$ (the functions evaluated at specific nodes $t$ and $s$ ).

3.2. Methodology As part of the technique, we shall take approximations of the barrier. Hence, given a function $f$, we consider a partition of the time domain $[0, t]$, namely $\Pi_{n}:=\left\{0=t_{0, n}<t_{1, n}<\cdots<t_{n, n}=t\right\}$. Then the approximating barrier is

$$
f_{n}(t):=\sum_{i=1}^{n} f\left(t_{i-1, n}\right) \mathbb{I}_{\left[t_{i-1, n}, t_{i, n}\right)}(t),
$$

where $\mathbb{I}$ is the indicator function. We take the partitions such that $\Pi_{m} \subset \Pi_{n}$ for $m<n$, and $\max _{0 \leq i, j \leq n}\left|t_{i, n}-t_{j, n}\right| \rightarrow 0$ as $n \rightarrow \infty$.

We have the following proposition.

Proposition 3.1. For any pair of nondecreasing functions $f$ and $g$ on $\mathbb{R}^{+}$with $f(0)>0$ and $g(0)>0$,

$$
P\left(T_{-g_{n}}^{f_{n}}<t\right) \rightarrow P\left(T_{-g}^{f}<t\right), \quad \forall t>0, \text { as } n \rightarrow \infty
$$

where $f_{n}$ and $g_{n}$ are the approximations as in (3.1) on the same partitions $\left\{\Pi_{n}, n=\right.$ $1,2, \ldots\}$.

PROOF. By construction $f_{n}(s) \leq f(s)$ and $g_{n}(s) \leq g(s)$ for all $s$. Thus, the sequence $P\left(T_{-g_{n}}^{f_{n}}<t\right), n=1,2, \ldots$ is decreasing, and is bounded from below by $P(T<t)$. Since we have $\left\{T_{-g_{n}}^{f_{n}}<t\right\} \rightarrow\left\{T_{-g}^{f}<t\right\}$ as $n \rightarrow \infty$, convergence holds.

Exploiting well known properties of the BM, in the following result we obtain an integral equation of Volterra type for the exit-time distribution. We use arguments similar to those in [10] or [13].

THEOREM 3.2. Let $f$ be a nondecreasing function such that $f(0)>0$. Then, the distribution $\Phi$ of the first exit time $T_{-f}^{f}(0)$ obeys the integral equation

$$
\begin{aligned}
\Phi(t)= & \int_{0}^{t} \varphi_{0}^{(-f(u), f(u))}(u) d u \\
& -\int_{0}^{t}\left(\int_{0}^{s} \varphi_{0}^{(-f(s)-f(u), f(s)-f(u))}(s-u) \Phi(d u)\right) d s
\end{aligned}
$$

where the function $\varphi_{x}^{(a, b)}(u)$ is given by (2.2). 
ProOf. Consider an approximation $\left\{f_{n}, n=1,2, \ldots\right\}$ for $f$ as in (3.1). We note

$$
\Phi_{n}(t)=P\left(T_{-f_{n}}^{f_{n}}<t\right)=\sum_{i=1}^{n} P\left(t_{i-1, n} \leq T_{-f_{n}}^{f_{n}}<t_{i, n}\right)
$$

Here, $\Phi_{n}(\cdot):=\Phi_{0}^{\left(-f_{n}, f_{n}\right)}(\cdot)$, and for simplicity we write $T_{-g}^{f}$ instead of $T_{-g}^{f}(0)$. By Proposition 3.1 we have that $\Phi_{n} \rightarrow \Phi$ as $n \rightarrow \infty$.

The proof is divided into three parts.

PART 1. We analyse the right-hand side of identity (3.3). For notational convenience, set $A_{i, n}=\left(t_{i-1, n} \leq T_{-f\left(t_{i-1, n}\right)}^{f\left(t_{i, 1}\right)}<t_{i, n}\right)$. Then for each term we observe that

$$
P\left(t_{i-1, n} \leq T_{-f_{n}}^{f_{n}}<t_{i, n}\right)=P\left(A_{i, n}\right)-P\left(A_{i, n}, T_{-f_{n}}^{f_{n}}<t_{i-1, n}\right)
$$

PART 2. The first part of the right-hand side of (3.4) can be obtained from (2.2) by integrating on the interval $\left[t_{i-1, n}, t_{i, n}\right)$. Hence, it reads as

$$
P\left(A_{i, n}\right)=\int_{t_{i-1, n}}^{t_{i, n}} \varphi_{0}^{\left(-f\left(t_{i-1, n}\right), f\left(t_{i-1, n}\right)\right)}(u) d u
$$

By the mean value theorem for integrals (see [2]) the previous equation becomes

$$
P\left(A_{i, n}\right)=\varphi_{0}^{\left(-f\left(t_{i-1, n}\right), f\left(t_{i-1, n}\right)\right)}\left(t_{i, n}^{*}\right)\left(t_{i, n}-t_{i-1, n}\right)
$$

for some value $t_{i, n}^{*} \in\left[t_{i-1, n}, t_{i, n}\right.$ ). Thus, in (3.3) we actually have a Riemann sum which converges to the desired quantity:

$$
\lim _{n \rightarrow \infty} \sum_{i=1}^{n} \phi_{0}^{\left(-f\left(t_{i-1, n}\right), f\left(t_{i-1, n}\right)\right)}\left(t_{i, n}^{*}\right)\left(t_{i, n}-t_{i-1, n}\right)=\int_{0}^{t} \varphi_{0}^{(-f(u), f(u))}(u) d u .
$$

Hence, the second element of (3.2) is obtained.

PART 3. For the last term in (3.4), we have the analysis

$$
\begin{aligned}
& P\left(A_{i, n}, T_{-f_{n}}^{f_{n}}<t_{i-1, n}\right) \\
& \quad=\int_{0}^{t_{i-1, n}} P\left(A_{i, n} \mid T_{-f_{n}}^{f_{n}}=u t\right) \Phi_{n}(d u) \\
& =\sum_{k=1}^{i-1} \int_{t_{k-1, n}}^{t_{k, n}} P\left(A_{i, n} \mid T_{-f_{n}}^{f_{n}}=u\right) \Phi_{n}(d u) .
\end{aligned}
$$


This in turn equals

$$
\begin{aligned}
& \sum_{k=1}^{i-1} \int_{t_{k-1, n}}^{t_{k, n}} P\left(A_{i, n} \mid T^{f_{n}}<T_{-f_{n}}, T_{-f_{n}}^{f_{n}}=u\right) \\
& \quad \times P\left(T^{f_{n}}<T_{-f_{n}} \mid T_{-f_{n}}^{f_{n}}=u\right) \Phi_{n}(d u) \\
& \quad+\sum_{k=1}^{i-1} \int_{t_{k-1, n}}^{t_{k, n}} P\left(A_{i, n} \mid T^{f_{n}} \geq T_{-f_{n}}, T_{-f_{n}}^{f_{n}}=u\right) \\
& \quad \times P\left(T^{f_{n}} \geq T_{-f_{n}} \mid T_{-f_{n}}^{f_{n}}=u\right) \Phi_{n}(d u) .
\end{aligned}
$$

Now, we analyse the quantities involved in the sums. We know that

$$
P\left(T^{f_{n}}<T_{-f_{n}} \mid T_{-f_{n}}^{f_{n}}=u\right)+P\left(T^{f_{n}} \geq T_{-f_{n}} \mid T_{-f_{n}}^{f_{n}}=u\right)=1
$$

By the symmetry of the BM,

$$
P\left(T^{f_{n}}<T_{-f_{n}} \mid T_{-f_{n}}^{f_{n}}=u\right)=P\left(T^{f_{n}} \geq T_{-f_{n}} \mid T_{-f_{n}}^{f_{n}}=u\right)=\frac{1}{2} .
$$

On the other hand, for each integral in the sums, let $u \in\left[t_{k-1, n}, t_{k, n}\right)$ with $k \leq i-1$. From the regenerative properties of the BM,

$$
\begin{aligned}
P\left(A_{i, n} \mid T^{f_{n}} \geq T_{-f_{n}}, T_{-f_{n}}^{f_{n}}=u\right) \\
=P\left(t_{i-1, n}-u \leq T_{-f\left(t_{i-1, n}\right)-f\left(t_{k-1, n}\right)}^{f\left(t_{i-1, n}\right)-f\left(t_{k-1, n}\right)}<t_{i, n}-u\right) \\
\quad=\int_{t_{i-1, n}-u}^{t_{i, n}-u} \varphi_{0}^{\left(-f\left(t_{i-1, n}-f\left(t_{k-1, n}\right), f\left(t_{i-1, n}\right)-f\left(t_{k-1, n}\right)\right)\right.}(w) d w .
\end{aligned}
$$

For this last step, the assumption of nondecreasing barriers is important. After a change of variable and application of the mean value theorem for each $k=1,2, \ldots, i-1$ the probability (3.7) becomes

$$
\varphi_{0}^{\left(-f\left(t_{i-1, n}\right)-f\left(t_{k-1, n}\right), f\left(t_{i-1, n}\right)-f\left(t_{k-1, n}\right)\right)}\left(t_{k, i, n}^{*}-u\right)\left(t_{k, n}-t_{k-1, n}\right),
$$

for some $t_{k, i, n}^{*} \in\left[t_{i-1, n}, t_{i, n}\right)$ and $k<i-1$ and $i=1,2, \ldots, n$.

Furthermore, for the last sums at (3.5), owing to the symmetry of the BM,

$$
P\left(A_{i, n} \mid T^{f_{n}}<T_{-f_{n}}, T_{-f_{n}}^{f_{n}}=u\right)=P\left(A_{i, n} \mid T^{f_{n}} \geq T_{-f_{n}}, T_{-f_{n}}^{f_{n}}=u\right) .
$$

So, using also (3.6) and (3.8), probability (3.5) ends up as

$2 \sum_{k=1}^{i-1} \int_{t_{k-1, n}}^{t_{k, n}} \varphi_{0}^{\left(-f\left(t_{i-1, n}\right)-f\left(t_{k-1, n}\right), f\left(t_{i-1, n}\right)-f\left(t_{k-1, n}\right)\right)}\left(t_{k, i, n}^{*}-u\right)\left(t_{k, n}-t_{k-1, n}\right) \frac{1}{2} \Phi_{n}(d u)$. 
Substitution in equations (3.4) and (3.3) yields

$$
\begin{aligned}
\sum_{i=1}^{n} & \sum_{k=1}^{i-1} \int_{t_{k-1, n}}^{t_{k, n}} \varphi_{0}^{\left(-f\left(t_{i-1, n}\right)-f\left(t_{k-1, n}\right), f\left(t_{i-1, n}\right)-f\left(t_{k-1, n}\right)\right)} \\
& \times\left(t_{k, i, n}^{*}-u\right)\left(t_{k, n}-t_{k-1, n}\right) \Phi_{n}(d u) .
\end{aligned}
$$

Upon applying again the mean value theorem, there are $t_{i, k, n}^{*} \in\left[t_{k-1, n}, t_{k, n}\right)$ for each $i<n$, such that sum (3.9) equals

$$
\begin{aligned}
\sum_{i=1}^{n} & \sum_{k=1}^{i-1} \varphi_{0}^{\left(-f\left(t_{i-1, n}\right)-f\left(t_{k-1, n}\right), f\left(t_{i-1, n}\right)-f\left(t_{k-1, n}\right)\right)}\left(t_{k, i, n}^{*}-t_{i, k, n}^{*}\right) \\
& \times\left(t_{k, n}-t_{k-1, n}\right)\left(\Phi_{n}\left(t_{i, n}\right)-\Phi_{n}\left(t_{i-1, n}\right)\right) .
\end{aligned}
$$

Equation (3.10) represents a Riemann-Stieltjes sum of a continuous function. Thus, when $n \rightarrow \infty$ we obtain the limit

$$
\int_{0}^{t}\left(\int_{0}^{s} \varphi_{0}^{(-f(s)-f(u), f(s)-f(u))}(s-u) \Phi(d u)\right) d s,
$$

which finally yields the last part of (3.2). This concludes the proof.

\section{Equations for the density}

In the previous section, Theorem 3.2 stated an integral equation for the distribution of $T$, the first exit time. There are results on first passage times requiring additional conditions in order to derive an integral equation for the density. In $[10,22,24]$ the barrier needs to be differentiable. In our case, we can readily see in (3.2) that $\Phi$ is differentiable, and thus we are able to obtain an integral equation for the density.

COROLlaRY 4.1. Under the conditions of Theorem 3.2, we have that the density of $T$, which we denote by $\varphi$, satisfies the integral equation

$$
\varphi(t)=\varphi_{0}^{(-f(t), f(t))}(t)-\int_{0}^{t} \varphi_{0}^{(-f(t)-f(u), f(t)-f(u))}(t-u) \varphi(u) d u,
$$

where the function $\varphi_{x}^{(a, b)}(u)$ is given by (2.2).

REMARK 1. As mentioned above, generally one assumes differentiable barriers to ensure the existence of a density. However, since the barriers we use are nondecreasing, one also has that the distribution function is differentiable.

REMARK 2. Since the function $\varphi_{x}^{(-a, b)}(u)$ is continuous in all its arguments, (4.1) has unique solution. This is a classical result in the theory of integral equations (see [6, Theorem 5, Page 183]). 
4.1. Another expression for the exit time density From the proof of Theorem 3.2, we can see that it is possible to modify the integral equation slightly. Recall the notation $T_{-g}^{f}(x)$; in (3.7),

$P\left(A_{i, n} \mid T^{f_{n}} \geq T_{-f_{n}}, T_{-f_{n}}^{f_{n}}=u\right)=P\left(t_{i-1, n}-u \leq T_{-f\left(t_{i-1, n}\right)}^{f\left(t_{i-1, n}\right)}\left(f\left(t_{k-1, n}\right)\right)<t_{i, n}-u\right)$

for $u \in\left(t_{k-1, n}, t_{k, n}\right]$. Finally this becomes

$$
\int_{t_{i-1, n}-u}^{t_{i, n}-u} \varphi_{f\left(t_{k-1, n}\right)}^{\left(-f\left(t_{i-1, n}\right), f\left(t_{i-1, n}\right)\right)}(w) d w .
$$

The idea is to consider a BM starting at $f\left(t_{k-1, n}\right)$, rather than at 0 , as was originally done in (3.7).

This change gives a new expression for the integral equation, namely

$$
\varphi(t)=\varphi_{0}^{(-f(t), f(t))}(t)-\int_{0}^{t} \varphi_{f(u)}^{(-f(t), f(t))}(t-u) \varphi(u) d u .
$$

4.2. Numerical solutions We now exploit a numerical procedure to solve (4.1). A numerical algorithm based on a recursive formulae is quite straightforward to implement; we summarize it briefly (the interested reader can refer to [17]).

We want to solve the Volterra integral equation

$$
F(t)=G(t)+\int_{0}^{t} K(t, s) F(s) d s,
$$

where $G$ and $K$ are known functions, the latter usually being called the kernel. Equation (4.2) is an equation of the second kind because function $G$ is nonzero, which is important for the method to work.

Suppose that we want to obtain an approximation of $F$ in the interval $[0, r]$, and we divide it into $N$ equally spaced subintervals of size $h$. We have $N+1$ nodes $\left\{t_{0}, t_{1}, \ldots, t_{N}\right\}$ such that $t_{n+1}-t_{n}=h, n=0,1, \ldots, N-1$, with $t_{0}=0$. We denote by $\left\{F_{1}, \ldots, F_{N}\right\}$ the approximation of $f$ at the nodes $\left\{t_{1}, \ldots, t_{N}\right\}$. The following recursive formula can be used:

$$
F_{n+1}=\frac{G\left(t_{n+1}\right)+\sum_{k=1}^{n} F_{k} \int_{t_{k-1}}^{t_{k}} K\left(t_{n+1}, s\right) d s}{1-\int_{t_{n}}^{t_{n+1}} K\left(t_{n+1}, s\right) d s}, \quad n=0, \ldots, N-1 .
$$

Notice that

$$
F_{1}=\frac{G(h)}{1-\int_{0}^{h} K(h, s) d s} .
$$

EXAmple 3. Using Scilab 4.1.2, we compute the density function of $T_{-1}^{1}(0)$ and $T_{-f}^{f}(0)$, where $f(t)=1+0.0001 t$. The two densities should be close to each other. In Figure 1 a sampling from the density of $T_{-1}^{1}(0)$ (determined by (2.2)) is shown as a line and similarly, the density of $T_{-f}^{f}(0)$ (determined by (4.3)) is shown with points. 


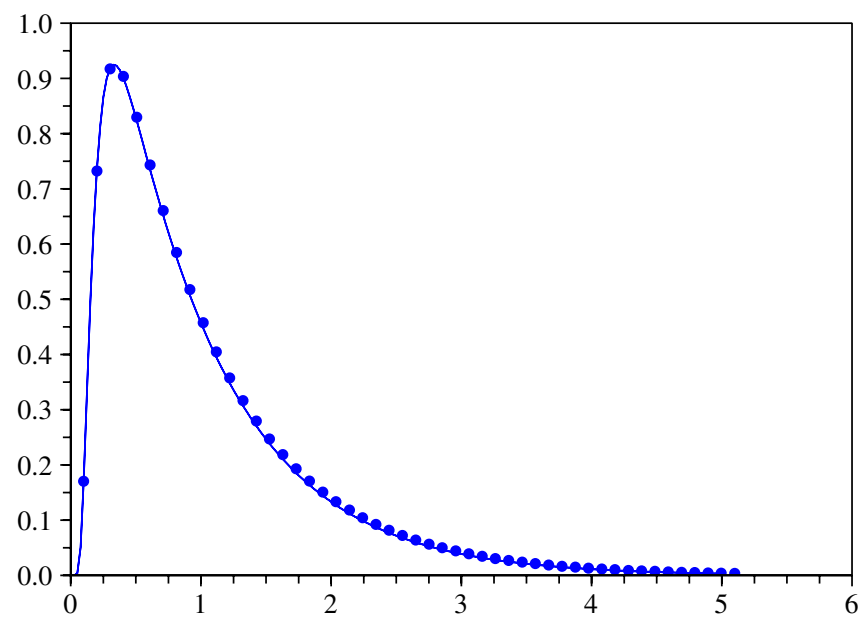

FIGURE 1. Densities for the exit times.

\section{Conclusion}

We studied the first-exit-time distribution of a reflected Brownian motion and found a Volterra integral equation for the density. The main result is derived from approximating the barriers by step functions and carrying out a careful analysis of the paths.

The solution of the integral equations does not seem a trivial task; however, it was shown to be feasible using numerical methods.

We briefly mention other possible directions to take.

- Extend the result to the case of nonsymmetric barriers. The main technical difficulty is the nonsymmetrical probabilities in (3.6).

- The problem of solving the integral equation explicitly remains open.

- There is an interesting relation between the maximum and the reflected Brownian motion. Let $M$ be the maximum of the Brownian motion, that is, $M_{t}=$ $\max \left(B_{s}, s \leq t\right)$. It is known the two processes,

$$
\left\{M_{t}-B_{t}: t \geq 0\right\} \quad \text { and } \quad\left\{\left|B_{t}\right|: t \geq 0\right\}
$$

have the same law (see, for example, [14, Page 210]). We may want to use this to find the first passage distribution of $M$.

\section{Acknowledgement}

This research was partially supported by the CONACYT-NSF (Mexico-USA) program. 


\section{References}

[1] D. Bañuelos, R. D. DeBlassie and R. Smits, "The first exit time of a planar Brownian motion from the interior of a parabola", Ann. Probab. 29 (2001) 882-901.

[2] R. G. Bartle, The elements of real analysis, 2nd edn (John Wiley \& Sons, New York, 1976).

[3] R. A. Betensky, "A boundary crossing probability for the Bessel process", Adv. Appl. Probab. 30 (1998) 807-830.

[4] A. N. Borodin and P. Salminen, Handbook of Brownian motion-facts and formulae (Birkhäuser, Basel, 1996).

[5] D. L. Burkholder, "Exit times of Brownian motion, harmonic majorization and Hardy spaces", Adv. Math. 26 (1977) 182-205.

[6] W. Cheney, Analysis for applied mathematics (Springer, New York, 2001).

[7] D. A. Darling and A. J. F. Siegert, "The first passage problem for a continuous Markov process", Ann. Math. Statist. 24 (1953) 624-639.

[8] R. D. DeBlassie, "Exit times from cones in $R^{n}$ of Brownian motion", Probab. Theory Related Fields 74 (1987) 1-29.

[9] R. D. DeBlassie, "The change of a long lifetime for Brownian motion in a horn-shape domain", Electron. Comm. Probab. 12 (2007) 134-139.

[10] V. De-la-Peña and G. Hernández-del-Valle, "First-passage densities of Brownian motion over a non-decreasing, right continuous barrier". Unpublished technical report, Department of Statistics, Columbia University, 2007.

[11] J. L. Doob, "Heuristic approach to the Kolmogorov-Smirnov theorems", Ann. Math. Statist. 20 (1949) 393-403.

[12] D. S. Grebenkov, "NMR survey of the reflected Brownian motion", Rev. Modern Phys. 79 (2006) 1077.

[13] G. Hernández-del-Valle, "First passage time densities of Brownian motion and applications to credit risk". Ph. D. Thesis, Department of Statistics, Columbia University, 2005.

[14] I. Karatzas and S. E. Shreve, Brownian motion and stochastic calculus (Springer, New York, 1998).

[15] S. Karlin and H. M. Taylor, A first course in stochastic processes (Academic Press, New York, 1975).

[16] F. Klebaner, Introduction to stochastic calculus with applications, 2nd edn (Imperial College Press, London, 2005).

[17] R. Kress, Numerical analysis (Springer, New York, 1998).

[18] P. Levitz, D. S. Grebenkov, M. Zinsmeister, K. M. Kolwankar and B. Sapoval, "Brownian flights over a fractal nest and first-passage statistics on irregular surfaces", Phys. Rev. Lett. 96 (2006) 180601.

[19] W. V. Li, "The first exit time of Brownian motion from a parabolic domain", Electron. Comm. Probab. 12 (2003) 134-139.

[20] W. V. Li, "The first exit time of Brownian motion from an unbounded convex domain", Ann. Probab. 31 (2003) 1078-1096.

[21] M. Lifshits and Z. Shi, "The first exit time of Brownian motion from a parabolic domain", Bernoulli 8 (2002) 745-765.

[22] G. Peskir, "On integral equations arising in the first-passage problem for Brownian motion", J. Integral Equations Appl. 14 (2001) 397-423.

[23] D. Revuz and M. Yor, Continuous Martingales and Brownian motion (Springer, Berlin, 1999).

[24] L. M. Ricciardi, L. Sacerdote and S. Sato, "On an integral equation for first-passage-time probability densities", J. Appl. Probab. 21 (1984) 302-314. 